\title{
Improvement of Connectivity in Mobile Ad-hoc Networks by Adding Static Nodes Based on A Realistic Mobility Model
}

\author{
Morteza Romoozi ${ }^{1}$, Hamideh Babaei $^{2}$ \\ ${ }^{1}$ Computer Engineering Department, Islamic Azad University, Kashan Branch \\ Kashan, Iran \\ ${ }^{2}$ Computer Engineering Department, Islamic Azad University, Naragh Branch \\ Naragh, Iran
}

\begin{abstract}
One of the ad hoc networks challenges is the connectivity problem coming from changeable and dynamic topology of networks nodes. Adding static nodes is a solution for this challenge. These nodes are added in some critical points in network environment where lack of mobile nodes is sensed in them. Many attempts have been made but in most of these studies no attention has been paid to network mobility model or the problem has been solved based on unrealistic mobility model such as Random waypoint. This article presents an algorithm for finding best positions of these nodes, using two approximation methods, genetic algorithm and artificial fish swarm algorithm. Both algorithms consider both deployment cost objective and connectivity efficiency objective in finding the positions. Simulation result shows that finding these critical points and adding static nodes in them can effect on performance of ad hoc networks.
\end{abstract}

Keywords: Ad-hoc networks, mobility model, connectivity, genetic algorithm, fish swarm algorithm.

\section{Introduction}

Ad hoc networks are networks without any fixed structure. In this network each of nodes has two roles: one is network mobile node and another role is transferring packet of other nodes. One of the main properties of this network is mobility of its nodes which causes network topology to change.

Mobility model is one of the most important parts of the adhoc network simulator which dictates the nodes the way of primary positioning and movement. Moreover, this model should be able to simulate a terrain that the node active in it. Consequently the closer model is to reality, the more reliable results of network evaluation are. In practice, evaluations that are done based on unrealistic mobility model can produce different results in comparison to simulation.

Connectivity in ad hoc network vary because of continues movement of nodes. It is possible that the movement of one or more nodes from one point to another causes the network partitioning, because each node plays the role of router in network. This makes connectivity to one of the network's main problems and many researchers are trying to improve the network connectivity by using different methods. But since the node movement is the main reason for partitioning, studies must be done by considering the node movements and mobility models. However an efficient method that is independent from other network parameters including routing protocol and signal propagation, is adding static nodes in critical points of the network.

Critical points are the points in which lack of nodes is more felt and adding static node in them creates the most amount of connection in comparison to other points. Furthermore in finding such point, deployment cost of static nodes must be considered. It means each point of network terrain has two objectives for selecting as position of static point, its efficiency for solving portioning problem and deployment cost.

This paper tries to find these points using two approximation algorithms, genetic algorithm and fish swarm algorithm.

The organization of this article is as follows: first the previous related works are reviewed. Then the mobility model and its relation to connectivity is presented, and later a realistic mobility model is introduce, finally two methods for adding static nodes based on this mobility model is suggested.

\section{Related Works}

In the section, we briefly review previous work related to the connectivity issue in wireless networks.

Gupta and Kumar showed in [1] that the critical common range $r_{n}$ for connectivity of $n$ randomly distributed wireless nodes in a disk of unit area satisfies that, if $\pi r_{n}^{2}=\frac{\ln n+c(n)}{n}$, 
then the resulting network is asymptotically connected with probability 1 if and only if $\mathrm{c}(\mathrm{n}) \rightarrow \infty$.

Ue and Kumar [2] studied the relationship between connectivity and node degree from another angle. They assumed the same number of nearest neighbors are maintained for each node, and showed that (i) the network is asymptotically disconnected with probability 1 as $n$ increases, if each node is connected to less than $0.074 \operatorname{logn}$ nearest neighbors; and (ii) the network is asymptotically connected with probability 1 as $n$ increases, if each node is connected to more than $5.1774 \operatorname{logn}$ nearest neighbors.

Wan and Yi [3] further studied the critical number of neighbors for k-connectivity and found the upper bound to be $\alpha e \log$ n, where $\alpha>1$ is a real number and $e \cong 2.718$ is the natural base. Khuller [4] studied the Connectivity Augmentation problem and determined a set of edges of minimum weight to be inserted so that the resulting graph is $\lambda$-vertex(edge)-connected. The problem is NP-hard for $\lambda>1$. Ausiello et al. [5] considered the Minimum GeometricDisk Cover (MGDC) problem. Given a s et of points $\mathrm{P}$ in the Euclidean plane and a rational number $r>0$, they intend to find the set of centers $\mathrm{C}$ with the minimum cardinality, such that every point in $\mathrm{P}$ is covered by a disk of radius $\mathrm{r}$ that is centered at one of the points in $\mathrm{C}$.

\section{Cluster Based Mobility Model}

There are different types of nodes in an ad-hoc networks that are active in it. Each type has a movement pattern that is represeted by mobility model in network simulators. Mobility model dictates to nodes how to move in their network environment. Many mobility models are proposed. Cluster Based Mobility Model $[6,7]$ has been proposed by authors previously.

In this model mobile nodes are grouped in several clusters as each cluster has several common characteristics such as speed, pause time, activity area and finding path or destination method. Activity area is some areas that probability the nodes existence in those areas is more than other places. This model can simulate environment obstacles and pathways. It means this model constrains the nodes to move in predefined pathways and their signals are obstructed by obstacle environments.

\section{Relation Between Connectivity and Mobility Model}

A simple way for solving the connectivity problem is to add static nodes in environment. In this way, if the mobile nodes of network leave a special area of network which causes network partitioning, then these static nodes play the role of these nodes as their support. These special areas are some points of network terrain where are more sparse than other points, therefore probability of partitioning in these points is more than other points. These points are called critical points.

This suggested solution has some problems, including too many adding static nodes in network first puts the nature of ad hoc network under question and second it is very costly. But we need limited number of static nodes in ad-hoc network. These limited static nodes just are for share internet, intranet or file server and so on.

But the question is how the mobility model of network nodes can be analyzed to find the critical points of network. To answer this question, a realistic mobility model can be used. This is because a realistic mobility model determines the realistic movement pattern of nodes and environment obstacles, hence by analyzing such model the position of the critical points can be located for adding static nodes. It means, if we predict that how the nodes move in the terrain and have information about environment obstacle, we can determine which points of the terrain can cause to partitioning of the networks.

\section{Adding Static Nodes for Improving Connectivity}

Adding static nodes should be in places where leads to the maximum of connectivity and should has minimum cost. This cost includes deployment cost and static node cost. But how can these points are found. The simplest way to solve this problem is to evaluate all the possible points in order to determine the best points for static node position. Such method has two problems.

1. Evaluating all points by consideration two objectives is a NP-Complete problem.

2. Evaluating each point is very time consuming. It is because computer simulation should be done to evaluate each solution.

Therefore first the problem 1 must be proven and then a solution must be found to solve it with consideration to the problem 2 .

\subsection{NP-Completeness Proof}

To prove that a problem is NP-Complete, it can be modeled to a known NP-Complete problem. 0-1 knapsack problem is used for this goal. In knapsack problem, there are $n$ kinds of items, 1 through $n$. Each kind of item $i$ has a value $v_{i}$ and a weight $w_{i}$. It usually is assumed that all values and weights are nonnegative. The maximum weight that can be carried in the bag is $W$. There are several types of knapsack problems which some of them such as fractional knapsack are solvable. But there is no polynomial solution for $0-1$ knapsack [8]. This problem can be formulated as a follow.

Maximize $\sum_{i=1}^{n} v_{i} x_{i}$ 
Subject to $\sum_{i=1}^{n} w_{i} x_{i}<W, \quad x_{i} \in\{0,1\}$

As it is mentioned, the problem is selecting $\mathrm{k}$ points from $\mathrm{n}$ points so that each of them has two objectives for selection, deployment cost $\left(\mathrm{c}_{\mathrm{i}}\right)$ and coverage efficiency $\left(\mathrm{e}_{\mathrm{i}}\right)$. We want to maximize connectivity but the cost increases by increasing number of the static nodes. Hence the maximum cost for adding the static nodes must be less than W. Therefore this problem can be formulated as 0-1 knapsack if $\mathrm{e}_{\mathrm{i}}$ is considered as $\mathrm{v}_{\mathrm{i}}$ and $\mathrm{w}_{\mathrm{i}}$ as $\mathrm{c}_{\mathrm{i}}$.

\subsection{Proposed Algorithm Based on Mobility Model}

As it is proven, finding best points based on two mentioned objectives is a N P-complete problem. Hence approximate algorithms are suitable for such problems.

Each approximation algorithm has a routine to evaluate each solution. But as it is mentioned, evaluating each solution is a time-consuming task due of simulation way. Hence some heuristics is needed to avoid simulation for evaluation. Therefore mobility model can be analyzed to find these heuristics.

Considering the mentioned mobility model, we realized that different node causes different mobility features. One of these features is nodes activity area. The existence of static nodes in these areas can be very defective because the mobile nodes in those areas are active and there is no need for static nodes. Therefore these areas can be deleted from the areas permitted for placing the static nodes.

On the other hand, obstacles prevent node signals from passing. Thus if a static node is located near an obstacle, it obstruct the great part of the node signal and this will prevent the node adding from producing a good result. In other words the area around the obstacles can be deleted from the permitted for placing the static nodes.

This paper presents two approximation algorithm for finding these points, genetic algorithms and fish swarm algorithm. Both of them use mentioned heuristic for evaluation of a solution.

\section{Genetic Algorithm and Finding Static Nodes Places}

Genetic algorithm [9] can be used to find the best place for the static nodes by considering the building location and node activity area. In this algorithm, the geographic points related to node activity area and buildings are certain from the beginning and search the whole terrain to find the points for static nodes.

\subsection{Problem Encoding}

As mentioned earlier the main problem is finding the place of static nodes. Therefore each chromosome is a sequence of 2D coordination of static nodes. Each gene is made up a point with horizontal and vertical axis. Number of these genes equals to number of required static points.

\subsection{Designing Fitness Function}

Voronoi Diagram [10] does evaluation task for every solution or chromosome. First coordination of obstacle and clusters activity areas are added to each solution which is allocation of static point's coordination. The result is given to computation procedure of Voronoi diagram. Procedure creates diagram then cells coordination of diagram are calculated. Cells coordination is given to another procedure and its area value is calculated with triangulation method. Area difference of these cells is evaluation parameter. The less the area differences cause to the better the solution.

Since the operations mentioned above including creating Voronoi diagram, extracting cells, and computing them have been done with standard methods which have been used at different uses in different research [11], therefore it does not seem necessary to explain them in details again.

\subsection{Proposed Algorithm}

The proposed genetic algorithm for finding the best order of static nodes is as follows:

1) Initial population is randomly selected from problem solution space. About $50 \mathrm{c}$ hromosomes are produced randomly. For each chromosome several coordination of static point are produced randomly.

2) The generation operations are applied on the population which is evaluated by fitness function.

3) Elitism: 10 percent out of whole chromosome with the best fitness remain for the next generation. This causes chromosome with appropriate fitness no to be destroyed accidentally.

4) Crossover: we use standard methods such as division from one point in our algorithm. The amount of combination operator contribution to in constructing next generation is considered 0.8 .

5) Mutation: the mutation operator discover new chromosome in genetic algorithm. In this suggested algorithm the mutation operator is applied in a random point with the small probability $\mathrm{p}_{\mathrm{m}}$ in order to add new chromosome to the new population.

6) The new population created by the operators above is reevaluated by fitness function.

7) The condition for the algorithm stopping is evaluated. If the condition is not met, the algorithm is transferred to step 2 again. In this implementation the algorithm repetitions to 200 generations with the same best chromosome have been considered. It means if the best chromosome does not change for 200 generation, algorithm stop and best solution is selected. 


\section{An Artificial Fish Swarm Algorithm for Finding Statick Nodes Positions}

Artificial Fish Swarm Intelligence Algorithm (AFSA) [12] is a swarm intelligence optimization algorithm. Fish usually stay in the place with a lot of food, so this algorithm simulates the behaviors of fish based on this characteristic to find the global optimum by optimizing local optimum. Below code describes how this algorithm works.

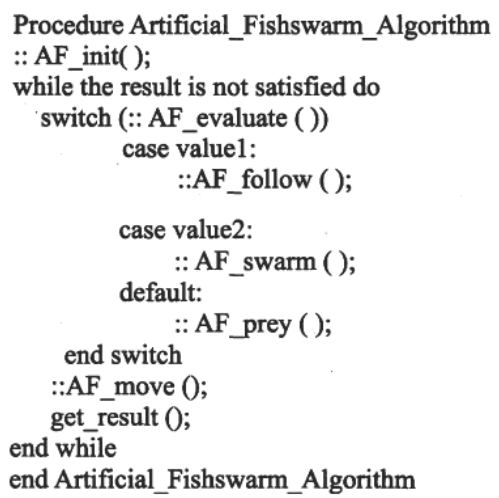

To describe this code, some definitions must be presented. These definitions are described as follow:

- AF: each Artificial Fish is considered as $\mathrm{X}=\left(\mathrm{n}, \mathrm{s}_{1}, \mathrm{~s}_{2}, \mathrm{~s}_{3}, \ldots, \mathrm{s}_{\mathrm{n}}\right)$ that $\mathrm{s}_{\mathrm{i}}$ is optimizing variable. In our positioning problem $\mathrm{x}_{\mathrm{i}}$ is considered as a static node position of the terrain which contain $(\mathrm{x}, \mathrm{y})$ coordination. Number of the static nodes is $\mathrm{n}$.

- Distance: distance between two fishes is indicated as $\mathrm{d}_{\mathrm{ij}}=\left\|\mathrm{X}_{\mathrm{i}}-\mathrm{X}_{\mathrm{j}}\right\|$. To calculate it, a Euclidean Minimum Spanning Tree is used. It means Euclidean minimum spanning trees for $X_{i}$ and $X_{j}$ are calculated. Each $\mathrm{x}_{\mathrm{i}}$ as a static node position, coordination of centers of activity area and coordination of centers of obstacles are assumed as vertices of a complete graph. In next step, spanning tree of this graph is calculated. Then total weights of both trees are calculated. Difference between total weight of tree of $X_{i}$ and $X_{j}$ is considered as distance between $X_{i}$ and $X_{j}$ or $d_{i j}$.

- Visual: Visual is the visual distance. Maximum of transmission range of each static node $\left(r_{s}\right)$ is assumed as Visual in this problem.

- Step: step is maximum step length of the fish. $2 * r_{s}$ is assumed as Step.

\subsection{AF Initialization}

In initialization step, population of AFs is formed. As it is mentioned, there is the cost constraint for number of the static points. $\mathrm{W}$ is assumed as maximum of budget for all static points. So this step generates random population that contain AFs which number of their variable is between $\mathrm{w} / \mathrm{c}_{\min }$ and $\mathrm{w} / \mathrm{c}_{\max } \cdot \mathrm{c}_{\min }$ and $\mathrm{c}_{\max }$ are minimum and maximum cost of adding a static point to the terrain. This cost is variable because of variable deployment cost. So to generate each $\mathrm{AF}, \mathrm{n}$ is a $\mathrm{r}$ andom number between $\mathrm{w} / \mathrm{c}_{\min }$ and $\mathrm{w} / \mathrm{c}_{\max }$ and each $\mathrm{s}_{\mathrm{i}}, \mathrm{i}=\{1, \mathrm{n}\}$ is generated randomly, ie $\mathrm{x}_{\mathrm{i}}, \mathrm{y}_{\mathrm{i}}$ take a random number between 0 and maximum of terrain size.

\subsection{AF Fitness (Food Consistency)}

Evaluation each AF must be capable to consider both cost and connectivity efficiency objective for evaluation an AF. For measuring connectivity efficiency, the mentioned mobility model heuristics are used. Therefore the network terrain should be divided into homogeneous cells with high connectivity. These cells should either include an activity area of cluster or have static node which guarantees their connectivity. Hence these cells can guarantee the connectivity of all parts of the terrain.

To do this Voronoi diagram can be used. In the simplest case, there are given a set of points $S$ in the plane, which are the Voronoi sites. Each site $s$ has a V oronoi cell, $V(s)$ consisting of all points closer to $s$ than to any other site.

Input points for Voronoi diagram are the building center, the node activity area center and static nodes positions that are extracted from the AF. If these points cause to make the Voronoi diagram with homogeneous cells, we can said that we distribute static nodes properly. Homogeneous cells are those cells that have same area and can cover the environment similarly. Therefore deference between areas of these cells is considered as connectivity efficiency objective.

Cost objective of each AF includes cost of static node and deployment cost of them that both of them must be considered. Equation 3,4 calculate cost objective.

$\mathrm{C}=\sum_{i=1}^{n} c_{s i}+c_{d i}$

$\mathrm{CF}=\mathrm{C}+\mathrm{D}$

$\mathrm{c}_{\mathrm{si}}$ is cost of ith static node and $\mathrm{c}_{\mathrm{di}}$ is deployment cost of ith static node which is extracted from al abeled map that indicates deployment cost of each region. FC is objective function of the $\mathrm{AF}$ or food consistence of it.

\subsection{AF Behaviors}

Four basic behaviors of AF are defined as follows: (1). AF_Prey: generally the fish perceives the concentration of food in water to determine the movement by vision or sense and then chooses the tendency. (2). AF_Swarm: The fish will assemble in groups naturally in the moving process, which is a kind of living habits. This behavior is done if colony is not so congested. (3). AF_Follow: In the moving process of the fish swarm, when a single fish or several ones find food, the neighborhood partners will trail and reach the food quickly. (4). AF_Move: Fish swim randomly in water; in fact, they are seeking food or companions in larger ranges. 
To explain this behavior briefly, below pseudo codes are presented.

AF Praying: $X_{i}$ is current state of the $A F$ and $X_{j}$ is a state in visual of the $\mathrm{AF}$ that is generated randomly (equation 5). If $\mathrm{FC}_{\mathrm{i}}<\mathrm{FC}_{\mathrm{j}}$ then $\mathrm{AF}$ moves toward $\mathrm{Xj}$ (equation 6). This process is done several times and if $\mathrm{AF}$ could not find a better state in its visual, a random state is selected (equation 7).

$\mathrm{X}_{\mathrm{j}}=\mathrm{X}_{\mathrm{i}}+\operatorname{Random}($ Visual$/ 2)$

$\mathrm{s}_{\mathrm{inext} \mathrm{k}}=\mathrm{s}_{\mathrm{ik}}+\operatorname{Random}(\operatorname{Step}) \frac{s_{j k}-s_{i k}}{\left\|X_{j}-X_{i}\right\|}$

$\mathrm{s}_{\mathrm{i} \mid \text { next } \mathrm{k}}=\mathrm{s}_{\mathrm{ik}}+$ Random(Step)

$\mathrm{s}_{\mathrm{ik}}$ is kth variable in $\mathrm{X}_{\mathrm{i}}$ and $\mathrm{s}_{\mathrm{jk}}-\mathrm{s}_{\mathrm{ik}}$ is Euclidean distance between $s_{j k}$ and $s_{i k}$.

AF Swarm: FCc is average FC of a swarm in visual of the $\mathrm{AF}$. AF move toward center of the swarm (equation 8), if $\mathrm{FCc} / \mathrm{n}_{\mathrm{f}}>\delta \mathrm{FC}_{\mathrm{i}}$, it means the swarm is not so crowded. $\delta$ is crowd factor which is between 0 and 1 . Otherwise the AF selects praying behavior.

$\mathrm{X}_{\mathrm{inext}}=\mathrm{X}_{\mathrm{ik}}+\operatorname{Random}(\mathrm{Step}) \frac{s_{c k}-s_{i k}}{\left\|X_{c}-X_{i}\right\|}$

AF Following: In following behavior, AF finds AF with maximum of $\mathrm{FC}$ in its vision. Then if around it is not so crowded $\left(\mathrm{FC}_{\max } / \mathrm{n}_{\mathrm{f}}>\delta \mathrm{FC}_{\mathrm{i}}\right)$ moves toward it (equation 9).

$$
\mathrm{X}_{\mathrm{i} \mid \mathrm{nextk}}=\mathrm{X}_{\mathrm{ik}}+\operatorname{Random}\left(\text { Step) } \frac{X_{\max }-X_{\mathrm{ik}}}{\left\|X_{\max }-X_{i}\right\|}\right.
$$

AF Evaluation (Choosing Bahavior):For next behavior of the AF, all mentioned behaviors are evaluated and a behavior with best result is selected. Best result is a behavior with minimum FC.

\section{Simulation}

The main purpose of any simulation done is usually to evaluate the suggested method and to compare it with the previous methods. However the presented method in this article is not comparable with the previous ones. Because none of them have not considered the connectivity issue based on a realistic mobility model.

In order to insure the accuracy of the suggested method, the authors of this paper have considered a sample terrain with scale 0.1 of the real terrain (which will be discussed later). We divided that terrain in to 100 points in grid form. Then we evaluated the location of static points on these points by simulation, the results of which were compared with the method independent from simulation. The results indicated that the suggested method independent from simulation leads to similar results when compared with simulation of all possible points. However as for time, simulation of possible points is much more time-consuming even with scale 0.1 and with one static node. It is because by increasing the number of static nodes their places get dependent on each other and the number of simulation increases exponentially.

\subsection{Simulation Metrics}

To evaluate affect a new method on network performance, two aspects were considered:

1. To understand the network topology characteristics that influenced by our method, we evaluate the following metrics:

Node Density: The average number of neighbors per node. Average Broken Links: The average number of broken links along the simulation.

2. To evaluate effect of our method on routing protocol parameter we evaluate following parameters:

Data Packet Reception: The number of data packets received at their intended destinations.

Control Packet Overhead: The number of network-layer control packet transmissions.

It seems necessary to mentioned we used AODV routing protocol [13] in our simulation.

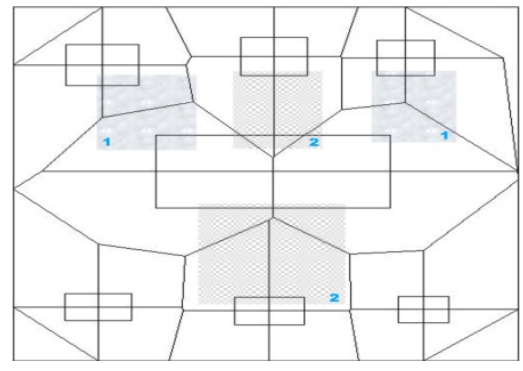

Fig. 1 Simulation terrain

\subsection{Simulation Parameters}

Figure1 shows the selected network terrain for evaluation the mobility model. It is assumed that there are two clusters of nodes with different activity area. In this figure the different activity area of two clusters are shown. Table1 indicate the simulation parameters. You can see simulator software, simulation terrain size, node transmission rang, signal propagation model, MAC layer protocol, bandwidth, speed range, pause time range, number of nodes respectively in this table. 
Table 1: Simulation Parameter

\begin{tabular}{|l|l|}
\hline Parameter & Value \\
\hline Simulator & $\begin{array}{c}\text { GlomoSim } \\
\text { simulator [14] }\end{array}$ \\
\hline Simulation terrain size & $1000 \mathrm{~m} \times 1000 \mathrm{~m}$ \\
\hline $\begin{array}{c}\text { Maximum node } \\
\text { transmission range }\end{array}$ & $250 \mathrm{~m}$ \\
\hline Propagation model & two-ray pathloss model \\
\hline MAC layer protocol & IEEE 802.11 DCF \\
\hline Bandwidth & $2 \mathrm{Mbps}$ \\
\hline Speed range & {$[0 \ldots 2 \mathrm{~m} / \mathrm{s}]$} \\
\hline Pause time range & {$[10 \ldots 300 \mathrm{~s}]$} \\
\hline Number of Nodes & 50 \\
\hline
\end{tabular}

Simulation was done in three followings ways:

- It was done by considering the obstacles and activity area of nodes for identifying static nodes points.

- It was done by considering activity area of nodes for identifying static nodes points without taking in to considering the obstacles both in mobility model and in calculating the location of static nodes (the obstacles have been deleted from the terrain)

- It was done without considering activity area and obstacles in calculating positions of static nodes points. It means that heuristics of mobility model does not considered and pays no attention to activity area and obstacles of terrain and just try to distribute static nodes in equal area cells.

Simulation for both proposed algorithm has been done separately. But found points by the both algorithms are near to each others. It means the both algorithms have the same behavior in finding these points. It can be result of the same evaluation method of the solutions in proposed algorithm. Therefore simulation results of the both methods have a little difference related to each other.

Therefore this result is produced by averaging between the points that produced by genetic algorithm and the points that produced with fish swarm algorithm.

\subsection{Simulation Results}

Node Density: The average number of node neighbors through the whole process simulations is shown in figure 2 . As it is shown in this figure the average number of node neighbors increases by an increases in the number of static nodes. This is because of the important connectivity and network node connectivity. That is because of that static nodes, node neighbor have increased. This increase can be seen in both diagrams. The interesting conclusion is that the improvement made are similar in both diagrams such similarity can prove that the suggested method is able to find the most suitable place for the static nodes by taking the obstacles in to consideration. Beside it can neutralize the effect of obstacles on positioning of static nodes.

It is sensible that if activity area and obstacle or mobility model heuristic has not considered the result be lower and lower node density has been earned. This matter can be because of effect of position of obstacles on signal of the nodes and effect of activity area of the nodes in placing static nodes. In fact by considering activity area of the nodes in finding position of static nodes, we can connect this activity area to each other.

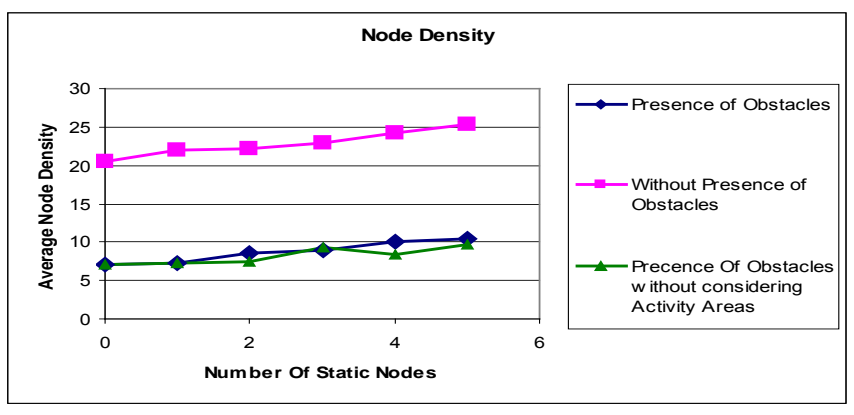

Fig. 2 Average Node Density

The average reception of data packet: The reception rate of data packet is shown in figure 3. As it is clear in figure 3, the average reception of data increases by an increase in the number of static nodes this was of course exactable. But why is not such increase considerable? Assuming the terrain size and number of nodes the result should not be changed to a great extent by adding a few number of static node with the transmission range similar to other nodes. But the encouraging result about the suggested method both diagrams have improved in a similar way. That is, no matter there is an obstacle or not, the improved remains the same. This is a significant issue because the suggested method can control the effect of obstacles in adding the static nodes and can provide condition similar to the state in which there is no obstacle.

Using of mobility heuristic in calculating position of static nodes, the results are better completely in comparison to when does not consider it because when we use this heuristic, different activity area can connect to each other by added static nodes and therefore more data packet can be received. 


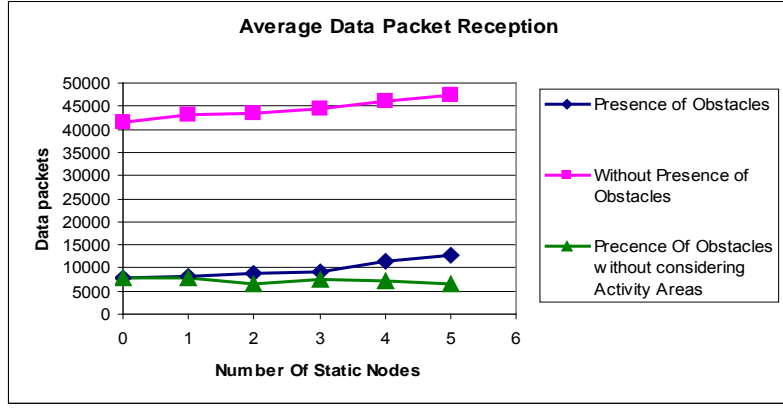

Fig. 3 Average Data Packet Reception

The average Control Packet Overhead: The number of control packets transmitted by increasing numbers of the static nodes has increased. This is because of increasing average data packet reception. More data packets need more control packet. As it illustrated in figure 4 Rate of increasing in both diagram has been kept.

While using mobility model heuristic produce higher results in data packet reception than does not using it but in some place of diagram equal result in control packet overhead has earned. It means by considering mobility heuristics we can create more strong links.

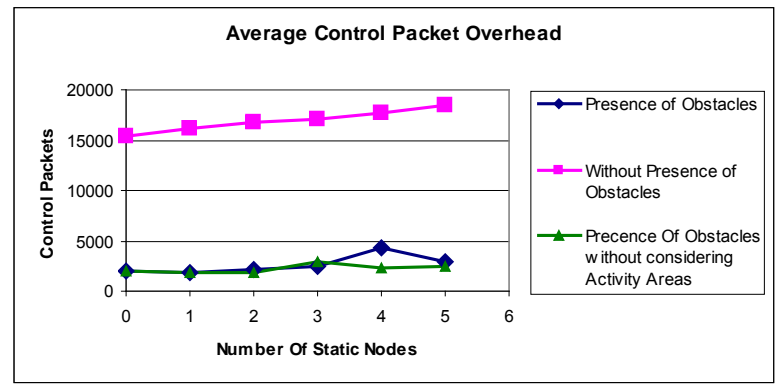

Fig. 4 Average Control Packet Overhead

The Average Broken Links: Figure 5 shows the Average Broken Links. Average broken links in both diagrams are almost constant while average data packet reception grows more than it. It means routs between the nodes by adding static nodes are stable. A little grows in broken links can be because of increasing the number of routs between the nodes but this routs have been broken because of movements of the nodes.

Number of broken links when has not been considered mobility model heuristic is less than when has been considered it. It can be because of more created routs between the nodes by connecting activity area to each other that have been broken by movement of the nodes.

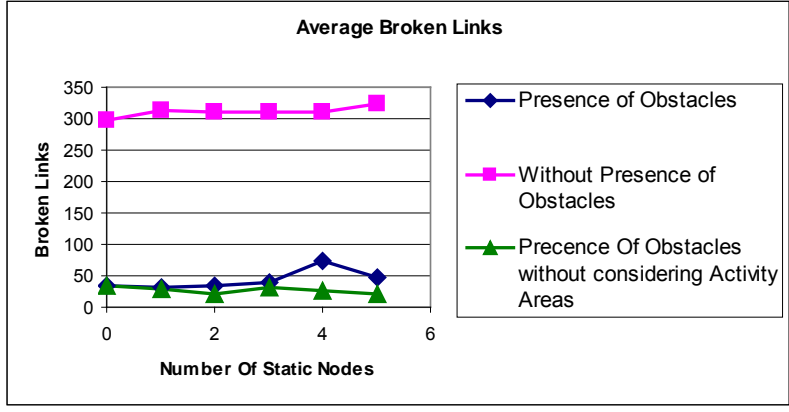

Fig. 5 Average Broken Links

\section{Conclusion}

In this paper we tried to improve the node connectivity issue by adding static nodes with consideration to deployment cost.

Connectivity improvement in unrealistic condition, without considering the terrain obstacles, can not be applied in realistic conditions. The realistic conditions for an ad hoc network are created by the realistic mobility model. Here in this article, connectivity and adding static nodes for improving it was evaluated based on a realistic mobility model presented by authors previously.

There are two important aspects of a mobility model that can be established in improvement of connectivity and finding static points. Hence the obstacles positions, because of blocking the signals of the nodes and the activity area, because of existence of the nodes in there have been considered.

Presented method can consider both connectivity efficiency objective and cost objective for finding critical points. This problem is a NP-complete problem.

The presented method can control the effect of obstacle on adding static nodes very well. As the result of simulation shows no matter there is an Obstacle or not the results of improvement rate are the same.

\section{References}

[1] P. Gupta, and P. R. Kumar, "Critical power for asymptotic connectivity in wireless networks" In Stochastic analysis, control, optimization and applications, 1998, Vol in honor of W. H. Fleming, pp 547-566.

[2] F. Xue, and P. R. Kumar, "The number of neighbors needed for connectivity of wireless networks", Wireless Networks, 2004, Vol.10(2), pp.169-181.

[3] P. J. Wan, and C. W. Yi, "Asymptotic critical transmission radius and critical neighbor number for k-connectivity in wireless ad hoc networks, 5th ACM Symposium on Mobile Ad Hoc Networking and Computing (MOBIHOC), 2004.

[4] S. Khuller, "Approximation algorithms for finding highly connected subgraphs", In D. S. Hochbaum, editor, Approximation algorithms for NP-hard problems. PWS Publishing Co., 1996.

[5] G. Ausiello, and P. Crescenzi, and G. Gambosi, and V. Kann, A. Marchetti-Spaccamela, and M. Protasi, "Complexity and 
IJ CSI International J ournal of Computer Science Issues, Vol. 8, Issue 4, No 2, July 2011

approximation: combinatorial optimization problems and their approximability properties", Springer-Verlag, Berlin, 1999.

[6] H. Babaei, and M. Fathi, and M. Romoozi, "Obstacle Mobility Model Based on Activity Area in Ad Hoc Networks", ICCSA, LNCS.2007, Vol.4706, pp. 804-817.

[7] M. Romoozi and H. Babaei, and M. Fathi, "A cluster-Based Mobility Model for Intelligent Nodes in Ad hoc Networks", ICCSA,LNCS, 2009, Vol.5592, pp. 804-817.

[8] T. H. Cormen, and C. E. Leiserson, and R. L. Rivest,and C. Stein, "Introduction to Algorithms" (3rd ed.), MIT Press.ISBN 0-262-03384-4, 2009.

[9] C. R. Reeves, and J. E. Rowe, "Genetic Algorithms Principles and erspectives A Guide to GA Theory (Operations Research/Computer Science Interfaces Series)", Vol. 20, pp.344., Hardcover ISBN: 978-1-4020-7240-6)

[10] F. Aurenhammer, "Voronoi Diagrams - A Survey of a Fundamental Geometric Data Structure", ACM Computing Surveys, 1991, Vol. 23(3), pp. 345-405.
[11] a. Bash, and j. Desnoyers, "Exact Distributed Voronoi Cell Computation in Sensor Networks", IPSN'07, 2007.

[12] X.L.Li, and Z.J.Shao, and J.X.Qian, "An optimizing method based on autonomous animals: fish-swarm algorithm", Systems Engineering Theory \& Practice, 2002, vol.22, no.11, pp. 32-38.

[13] C. E. Perkins, and E. M. Belding-Royer, "Ad Hoc On Demand Distance Vector (AODV) Routing", 2nd IEEE Workshop on Mobile Computing Systems and Applications, 1999, pp. 90-100.

[14] L. Bajaj, and M. Takai, and R. Ahuja, and K. Tang, and R. Bagrodia, and M. Gerla, "GlomoSim: A Scalable Network Simulation Environment", Technical Report CSD, \#990027, UCLA, 1997. 